\title{
Stress relaxation through interfacial sliding in nanocrystalline films
}

\author{
N V Skiba, I A Ovid'ko and A G Sheinerman \\ Institute of Problems of Mechanical Engineering, Russian Academy of Sciences, Bolshoj 61, \\ Vasiljevskii Ostrov, St Petersburg 199178, Russia \\ E-mail: ovidko@def.ipme.ru
}

Received 20 April 2008, in final form 9 September 2008

Published 13 October 2008

Online at stacks.iop.org/JPhysCM/20/455212

\begin{abstract}
A special mechanism of stress relaxation in nanocrystalline films is suggested and theoretically described. The mechanism represents the interfacial sliding accompanied by the formation of wedge disclination dipoles at grain boundaries in nanocrystalline films. The wedge disclination dipoles release, in part, mismatch stresses generated at film-substrate boundaries. It is theoretically shown that the special relaxation mechanism is energetically favorable in various nanocrystalline films deposited onto single crystalline substrates (in particular, AlN/6H-SiC, $\mathrm{GaN} / 6 \mathrm{H}-\mathrm{SiC}, 3 \mathrm{C}-\mathrm{SiC} / \mathrm{Si}$ and $\mathrm{Ni} / \mathrm{Cu}$ film/substrate systems) in wide ranges of their parameters.
\end{abstract}

\section{Introduction}

Defects and stresses in solid films represent a subject of intensive experimental and theoretical research efforts in condensed matter physics; see, e.g., [1-10]. In recent years, rapidly growing attention has been paid to nanocrystalline films consisting of crystalline grains with sizes lower than $100 \mathrm{~nm}$ and showing outstanding physical and mechanical properties; see, e.g., [11-15]. Such films can be divided into nanocrystalline thin films and thicker ones, often called nanocrystalline coatings. Both the structure and the properties of nanocrystalline films are significantly influenced by internal stresses occurring at interphase boundaries (first of all, boundaries between substrates and films) due to crystal lattice parameter mismatch, elastic modulus mismatch, thermal coefficient of expansion mismatch, and plastic flow mismatch between adjacent phases [16]. Commonly it is desired that the level of internal stresses be decreased, because these stresses are capable of initiating cracks and/or causing the structural transformations responsible for degradation of the functional characteristics of films. In several examples, it was experimentally detected that relaxation of the mismatch stresses in nanocrystalline films is enhanced compared to their conventional coarse-grained counterparts [17], and the level of residual stresses decreases with decreasing grain size in the nanoscale range [18]. For instance, as was noted in paper [17], residual stresses are low in nanocrystalline cermet coatings (thick films), resulting in the capability to produce very thick coatings. There, nanocrystalline coatings were fabricated up to $0.65 \mathrm{~cm}$ thick and could probably be made with arbitrary thickness [17]. At the same time, in a conventional polycrystalline cermet coating, stress buildup limits coating thickness to typically $500-800 \mu \mathrm{m}$. This enhancement of mismatch stress relaxation can be naturally attributed to the nanocrystalline effects causing the effective action of special stress relaxation mechanisms which are inherent only to nanocrystalline films and do not operate in conventional coarse-grained polycrystalline and single crystalline films. The main aim of this paper is to suggest and theoretically describe a special mechanism of stress relaxation in nanocrystalline films. The mechanism represents interfacial sliding accompanied by the formation of wedge disclination dipoles at grain boundaries in a nanocrystalline film.

\section{Interfacial sliding in nanocrystalline films. Basic features}

In general, in parallel with the standard lattice dislocation slip, specific deformation mechanisms conducted by interfaces significantly, or even crucially, contribute to plastic flow in nanocrystalline solids; see, e.g., reviews [19, 20] and book [21]. Such mechanisms-interfacial sliding, grain boundary diffusional creep and rotational deformationeffectively operate in nanocrystalline solids, first of all, due to the presence of a very large number of grain boundaries [19-21]. In the context discussed, it is natural to think that these specific deformation mechanisms can contribute to the plastic relaxation of mismatch stresses in 


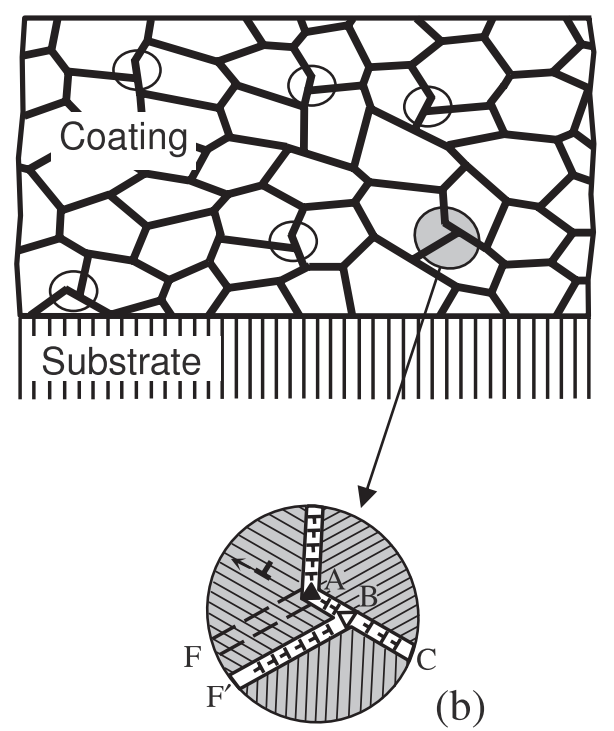

Figure 1. Nanocrystalline film deposited onto a single crystalline substrate. Interfacial sliding causes the formation of dipoles of disclinations (triangles). These disclination dipoles serve as sources of stress fields compensating for, in part, mismatch stresses in nanocrystalline films. (a) General view. (b) Magnified inset highlights the formation of wedge disclination dipoles due to interfacial sliding.

nanocrystalline films, in contrast to conventional coarsegrained or single crystalline films where the formation and slip of lattice misfit dislocations represent the dominant deformation mode causing stress relaxation. In particular, we think that the interfacial sliding-one of specific plastic deformation mechanisms intensively operating in nanocrystalline solids with the finest grains [19-26] - can effectively contribute to stress relaxation in nanocrystalline films. More precisely, the interfacial sliding leads to the formation of wedge disclination dipoles at grain and interphase boundaries [27, 28] (figure 1), and the disclination dipoles with certain geometric parameters release, in part, mismatch stresses in nanocrystalline films.

Let us briefly consider the geometric aspects concerning generation of wedge disclination dipoles due to interfacial sliding. Following [27, 28], interfacial sliding across a triple junction of interfaces produces a disclination dipole at an interface fragment along which the triple junction shifts (figure 1(b)). As to details, interfacial sliding along a grain boundary $\mathrm{AC}$ results in the displacement of both the triple junction $\mathrm{A}$ to its new position $\mathrm{B}$ and the grain boundary AF to its position $\mathrm{BF}^{\prime}$ (figure 1(b)). The grain boundary AF is assumed to be a symmetric tilt boundary characterized by a tilt misorientation parameter $\omega$. Interfacial sliding is, in part, accommodated by the emission of lattice dislocations into the left upper grain (figure 1(b)). When the triple junction is shifted by interfacial sliding from its initial position $\mathrm{A}$ to the position B (figure 1(b)), angle gaps $-\omega$ and $\omega$ appear at the grain boundary junctions A and B, respectively [27, 28]. According to the theory of defects in solids [29, 30], the junctions A and $\mathrm{B}$ with angle gaps $\pm \omega$ are considered as partial wedge disclinations with strengths $\pm \omega$ (for details, see $[27,28]$ ). (a)
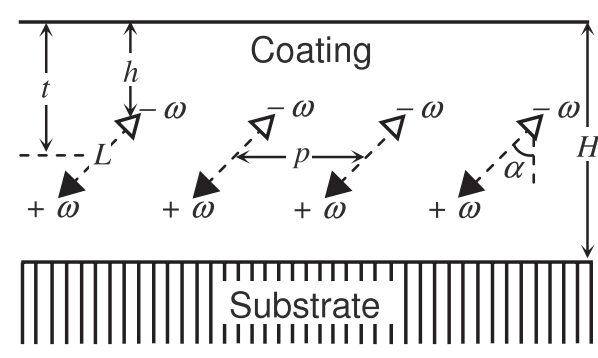

(b)

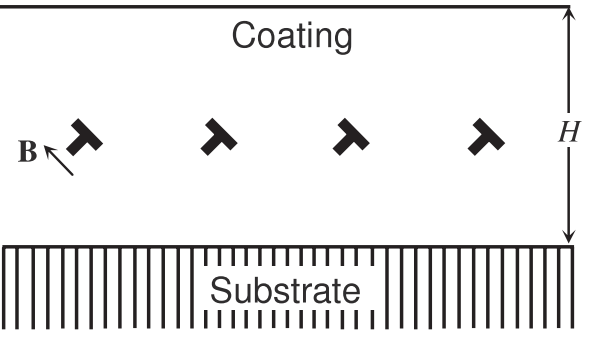

Figure 2. A model film/substrate system with a periodic row of misfit defects. (a) An array of wedge disclination dipoles. (b) An array of dislocations that create the same long-range stresses as disclination dipoles in (a) (for details, see the text).

The disclinations at the junctions $\mathrm{A}$ and $\mathrm{B}$ form a dipole configuration whose stress fields can compensate for, in part, mismatch stresses in nanocrystalline films.

Note that the formation of disclination dipoles due to the interfacial sliding (figure 1) occurs at triple junctions of grain boundaries, whose number is very large in the nanocrystalline matter. At the same time, the density of such triple junctions in conventional coarse-grained polycrystalline films is low, and triple junctions are absent in single crystalline films. In this context, interfacial sliding as a stress relaxation mechanism can operate in nanocrystalline films, but does not significantly contribute to stress relaxation in conventional coarse-grained and single crystalline films.

Also, note that disclination dipoles were experimentally observed by high resolution transmission electron microscopy (HRTEM) in iron under ball-mill treatment resulting in the nanostructure formation [31]. Besides, disclinations, their dipoles and other disclination configurations at grain boundaries were experimentally identified by various techniques in deformed coarse-grained polycrystals; see reviews [32, 33] and references therein.

\section{Energy characteristics of the formation of wedge disclination dipoles due to interfacial sliding in nanocrystalline films}

In order to reveal if interfacial sliding accompanied by the formation of wedge disclination dipoles (figure 1) can effectively release mismatch stresses in nanocrystalline films, we will calculate the energy characteristics of this formation process in the model film-substrate system shown in figure 2(a). The system is composed of a semi-infinite substrate and a film of thickness $H$ (figure 2(a)). The substrate and film in the model system are assumed to be elastically isotropic 
solids having the same values of the shear modulus $G$ and the same values of Poisson's ratio $v$. The film/substrate boundary creates mismatch stresses.

In general, one can distinguish three basic sources of misfit stresses in nanocrystalline films. First, in-plane misfit stresses in a film are generated by the film-substrate interface due to a difference (dilatational misfit) of the crystal lattice parameters $a_{\mathrm{f}}$ and $a_{\mathrm{s}}$ of the film and substrate, respectively. This difference is characterized by the dilatational misfit parameter $f=\left(a_{\mathrm{f}}-a_{\mathrm{s}}\right) / a_{\mathrm{f}}$. The existence of the dilatational misfit is generic in any film/substrate system (containing either single crystalline or polycrystalline or nanocrystalline film), because the materials of the substrate and the film are different by definition. Second, misorientation misfit stresses in a nanocrystalline film/substrate system are generated by the film-substrate interface due to the difference in the crystallographic orientations of the substrate and film nanograins. In doing so, the interface serves as a kind of grain boundary. Third, misorientation misfit stresses in a nanocrystalline film are generated by grain boundaries due to the difference in the crystallographic orientations of neighboring nanograins matched at these boundaries. The misorientation misfit stresses, created by the grain boundaries and the film-substrate interface, are short range. In fact, the misorientation misfit stresses of a grain boundary are created by the so-called equilibrium grain boundary dislocations that are arranged periodically with a short period and carry misorientation of the boundary; for details, see [34]. The stresses rapidly fall with the distance from the grain boundary. More precisely, the misorientation stress level is close to zero at the points distant by the equilibrium dislocation structure period or more from the boundary plane [34].

In general, grain boundaries and film-substrate interfaces can contain non-equilibrium defects that create long-range stresses $[21,34,35]$. In the case of nanocrystalline films, nonequilibrium defects at grain boundaries are naturally treated as misfit defects (analogs of misfit dislocations at film-substrate interfaces in single crystalline films) releasing, in part, both dilatational and misorientation stresses. In this paper, we focus our consideration on the specific type of misfit defects, namely, on the disclination dipoles generated at grain boundaries due to interfacial sliding.

Also, note that, since the misorientation stresses are short-range ones, the in-plane misfit stresses of the dilatation origin provide the dominant effect on possible initiation of cracks and/or structural transformations responsible for the degradation of functional characteristics of nanocrystalline films. In this context, in order to catch the main features of the problem examined, we consider the dilatational misfit $f$ as the only stress source in the nanocrystalline film-substrate system.

Let us examine the relaxation of dilatational misfit stresses (hereinafter called misfit stresses) due to grain boundary sliding in the nanocrystalline film. The interface-slidingproduced dipoles of wedge disclinations are assumed to be arranged periodically with a period $p$ (figure 2(a)). All the disclination dipoles are supposed to be identical. The distance between the disclinations of each dipole or, in other terms, the disclination dipole size is $L$. The strengths of the disclinations composing a dipole are $\pm \omega$. The angle $\alpha$ characterizes the orientation of each dipole relative to the film-substrate boundary as shown in figure 2(a). Although this model arrangement of disclination dipoles (figure 2(a)) is rather simple, it captures the essential physics of stress relaxation through interfacial sliding in real nanocrystalline films.

The role of interfacial-sliding-produced disclination dipoles in stress relaxation in films is illustrated by the fact that the array of disclination dipoles (figure 2(a)) is topologically equivalent to an array of dislocations (figure 2(b)) with the Burgers vectors determined by the disclination strength and the distance between the two disclinations of each dipole. More precisely, following the theory of disclinations [29, 30], a disclination dipole with disclination strengths $\pm \omega$ and a distance $L$ between the disclinations serves as a source of long-range stresses which is equivalent to a (super)dislocation with the Burgers vector magnitude $B=2 L \tan (\omega / 2)$. At the same time, the stress distribution in the area between the disclinations of a dipole configuration is different from that in the vicinity of its equivalent (super)dislocation $[29,30]$. In this context, the array of disclination dipoles (figure 2(a)) and the array of dislocations (figure 2(b)) cause similar (but not the same) effects in relieving the mismatch stresses in films.

Formation of the model ensemble of wedge disclination dipoles due to interfacial sliding in a film is characterized by the energy difference $\Delta W$ (per unit area of the film section parallel to the film surface) between the final (figure 2(a)) and initial (defect-free) states. Formation of the wedge disclination dipoles is energetically favorable, if $\Delta W<0$, and unfavorable otherwise. The energy difference $\Delta W$ has the following three basic terms:

$$
\Delta W=\frac{1}{p}\left(E_{\mathrm{self}}^{\Delta}+E_{\mathrm{int}}^{\Delta-f}+E_{\mathrm{int}}^{\Delta-\Delta}\right),
$$

where $E_{\text {self }}^{\Delta}$ is the proper energy of an individual wedge disclination dipole (per unit disclination length) (figure 2(a)); $E_{\text {int }}^{\Delta-f}$ is the energy that characterizes the interaction between an individual disclination dipole and the mismatch stresses (per unit disclination length), and $E_{\text {int }}^{\Delta-\Delta}$ is the energy that characterizes the interaction between the disclination dipoles (per unit disclination length and per dipole). In general, a Peierls-like barrier to the interfacial sliding contributes to the energy difference $\Delta W$. However, in the case under consideration, the interfacial sliding occurs in parallel with the film deposition onto a substrate at high-temperature conditions that enhance the interfacial sliding. In these circumstances, the Peierls-like barrier to the interfacial sliding at high-temperature conditions is negligibly low [36] and, for simplicity, is not taken into account in formula (1).

The proper energy $E_{\text {self }}^{\Delta}$ of a wedge disclination dipole is given by the standard formula [29]:

$$
E_{\mathrm{self}}^{\Delta}=\frac{D \omega^{2} L^{2}}{2}\left(\cos ^{2} \alpha+\frac{1}{2} \ln \frac{L^{2} \sin ^{2} \alpha+4 t^{2}}{L^{2}}\right),
$$

where $D=G /[2 \pi(1-v)], G$ is the shear modulus, $v$ denotes the Poisson ratio, as indicated above, and $t=h+(L / 2) \cos \alpha$ 


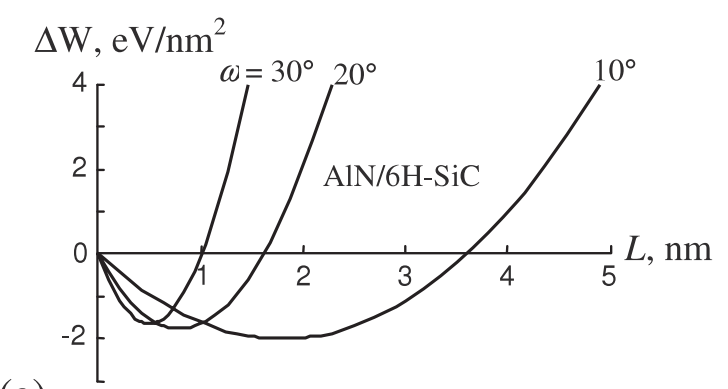

(a)

(c)
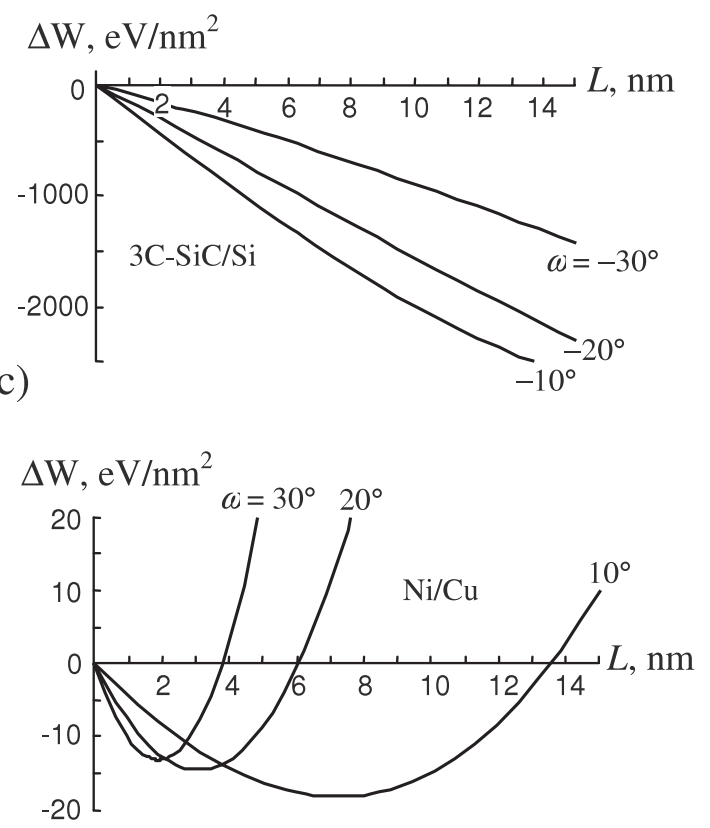

(d)

Figure 3. Dependences of energy difference $\Delta W$ on disclination dipole size $L$, for various values of disclination strength $\omega$ and film thickness $H=30 \mathrm{~nm}$, in film/substrate systems (a) AlN/6H-SiC, (b) GaN/6H-SiC, (c) 3C-SiC/Si and (d) Ni/Cu.

is the distance between the film-free surface and the line crossing the centers of the disclination dipoles (figure 2(a)). Following [4], the energy $E_{\text {int }}^{\Delta-f}$ of the interaction between a disclination dipole and the mismatch stresses is given as:

$$
E_{\text {int }}^{\Delta-f}=-4 \pi D(1+v) f \omega L t \cos \alpha .
$$

The energy $E_{\text {int }}^{\Delta-\Delta}$ of the interaction between the disclination dipoles is calculated using the standard formula [29] that describes the energy of the interaction of two disclinations in a semi-infinite isotropic solid. Performing the summation over all pairs of disclinations, we find:

$$
\begin{aligned}
E_{\mathrm{int}}^{\Delta-\Delta} & =D \omega^{2} \sum_{i=1}^{4} \sum_{j=0}^{\infty}(-1)^{i-1} \\
& \times\left(h_{i} h_{i}^{\prime}+\frac{\left(h_{i}-h_{i}^{\prime}\right)^{2}+\left(j p+p_{i}\right)}{4}\right. \\
& \left.\times \ln \frac{\left(h_{i}-h_{i}^{\prime}\right)^{2}+\left(j p+p_{i}\right)}{\left(h_{i}+h_{i}^{\prime}\right)^{2}+\left(j p+p_{i}\right)}\right)
\end{aligned}
$$

where $h_{1}=h_{2}=h_{1}^{\prime}=h_{4}^{\prime}=h ; h_{3}=h_{4}=h_{2}^{\prime}=h_{3}^{\prime}=$ $h+L \cos \alpha ; p_{1}=p_{3}=0 ; p_{2,4}= \pm L \sin \alpha$. Formulae (1)-(4) allow one to calculate the characteristic energy difference $\Delta W$.

\section{Results of model calculations in example cases of nanocrystalline film/substrate systems AlN/6H-SiC, GaN/6H-SiC, 3C-SiC/Si and Ni/Cu}

Let us calculate the characteristic energy difference $\Delta W$ in example cases of scientifically and practically important [37-44] nanocrystalline films of $\mathrm{AlN}, \mathrm{GaN}, 3 \mathrm{C}-\mathrm{SiC}$ and $\mathrm{Ni}$. We consider the following film/substrate systems: AlN/6H-SiC, $\mathrm{GaN} / 6 \mathrm{H}-\mathrm{SiC}, 3 \mathrm{C}-\mathrm{SiC} / \mathrm{Si}$ and $\mathrm{Ni} / \mathrm{Cu}$. In our consideration, we will assume that disclination dipoles form in films far enough from the film/substrate interface. In this case, the elastic moduli of substrates weakly influence the energies associated with the formation of disclination dipoles. Therefore, in a first approximation, we will use the film elastic moduli as the effective elastic moduli of the above film-substrate systems. Taking into account this approximation, the film/substrate systems are characterized by the following material parameters: $G=131 \mathrm{GPa}, v=0.287$ [45], $f=0.009$ [46] for AlN/6H$\mathrm{SiC} ; G=67 \mathrm{GPa}, v=0.352$ [45], $f=-0.0357$ [47] for $\mathrm{GaN} / 6 \mathrm{H}-\mathrm{SiC} ; G=217 \mathrm{GPa}, v=0.23$ [48], $f=-0.2$ [49] for $3 \mathrm{C}-\mathrm{SiC} / \mathrm{Si}$; $G=73 \mathrm{GPa}, v=0.4$ [50], $f=0.026$ [51], for $\mathrm{Ni} / \mathrm{Cu}$.

With these material parameters and formulae (1)-(4), we calculated the dependences of the energy difference $L$ on the key parameters of the model film/substrate system (shown in figure 2(a)) containing disclination dipoles. Figure 3 presents the dependences of the energy $\Delta W$ on the dipole size $L$, for AlN/6H-SiC (figure 3(a)), GaN/6H-SiC (figure 3(b)), 3C$\mathrm{SiC} / \mathrm{Si}$ (figure 3(c)) and $\mathrm{Ni} / \mathrm{Cu}$ (figure 3(d)), for various values of the disclination strength $\omega$. In doing so, in our calculations, we have used the following values for the other parameters of the defect structure (figure 2(a)): $\alpha=0^{\circ}, p=30 \mathrm{~nm}$, $H=30 \mathrm{~nm}$, and $h=H / 2$. As it follows from figures 3(a), (b) and (d), in the case of AlN/6H-SiC, GaN/6H-SiC and Ni/Cu, the dependences $\Delta W(L)$ have minimums corresponding to the equilibrium positions of the disclination dipoles. Each of these equilibrium positions is characterized by the equilibrium size $L_{\mathrm{e}}$ of the disclination dipoles. For the film/substrate system 3C-SiC/Si (figure 3(c)), $\Delta W$ is a monotonously decreasing function of $L$ at any $\omega$. Such monotonously decreasing behavior of $\Delta W(L)$, in the framework of our model (figure 2(a)), indicates that expansion (increase in $L$ ) 

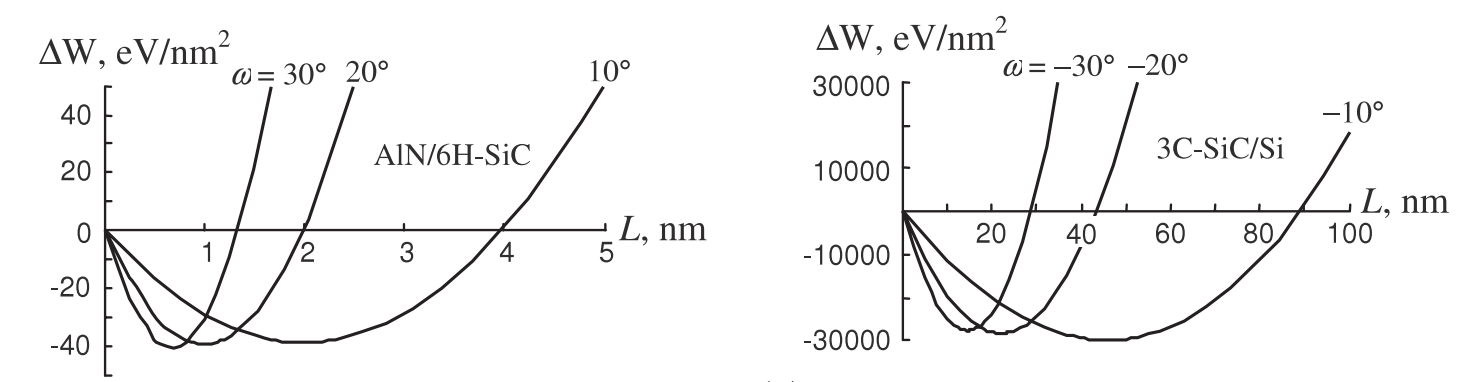

(a)

(c)

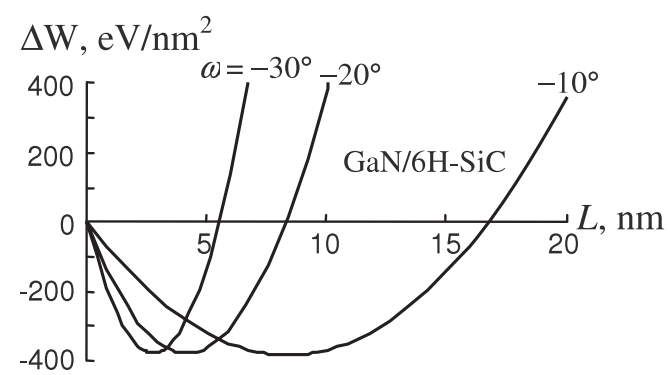

(b)

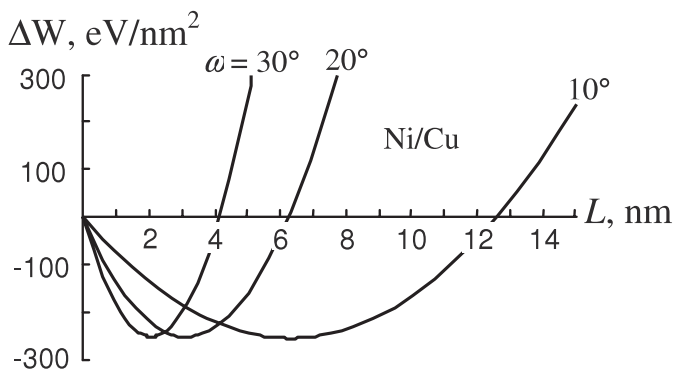

(d)

Figure 4. Dependences of energy difference $\Delta W$ on disclination dipole size $L$, for various values of disclination strength $\omega$ and film thickness $H=500 \mathrm{~nm}$, in film/substrate systems (a) AlN/6H-SiC, (b) GaN/6H-SiC, (c) $3 \mathrm{C}-\mathrm{SiC} / \mathrm{Si}$ and (d) Ni/Cu.
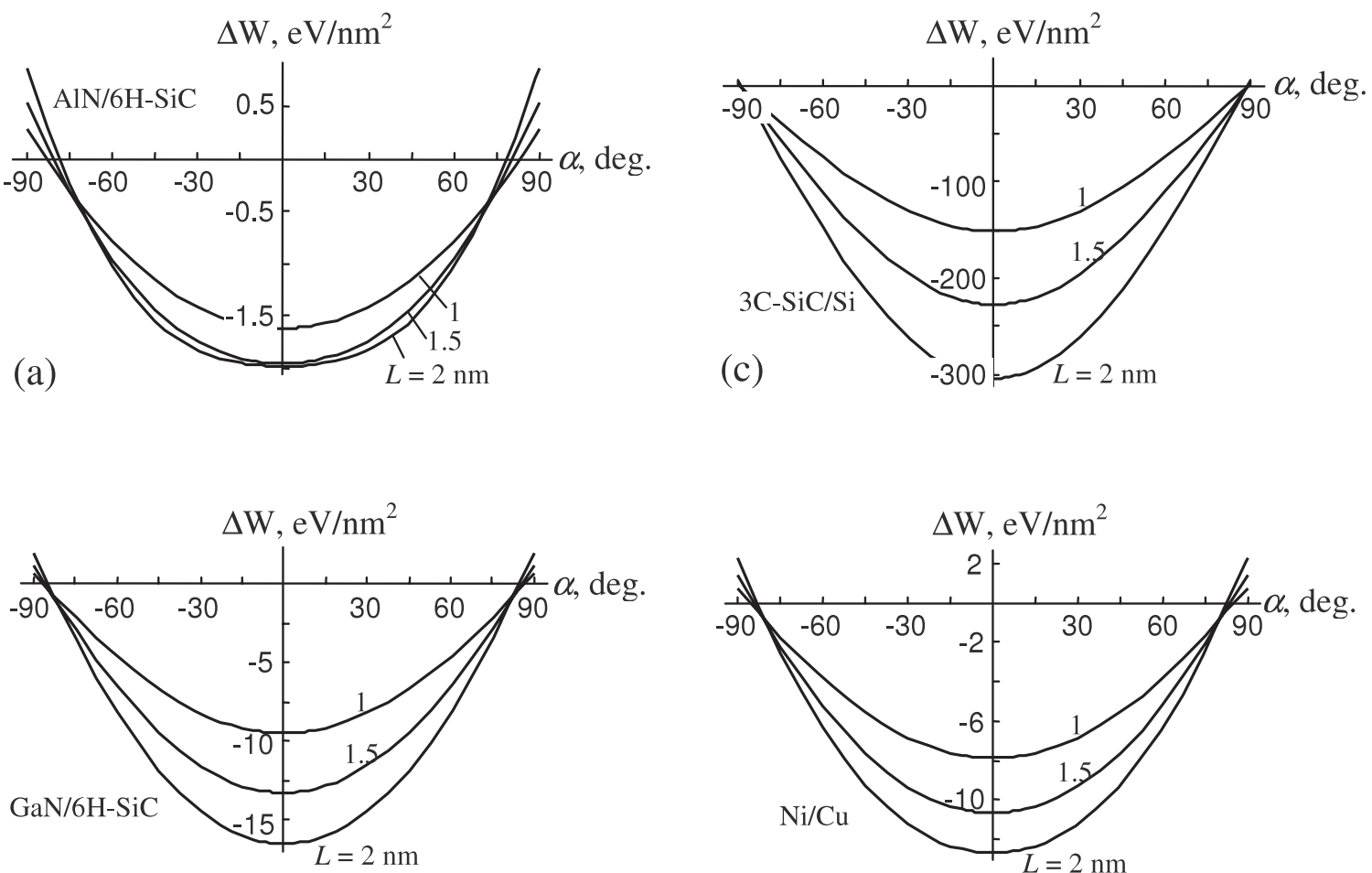

(b)

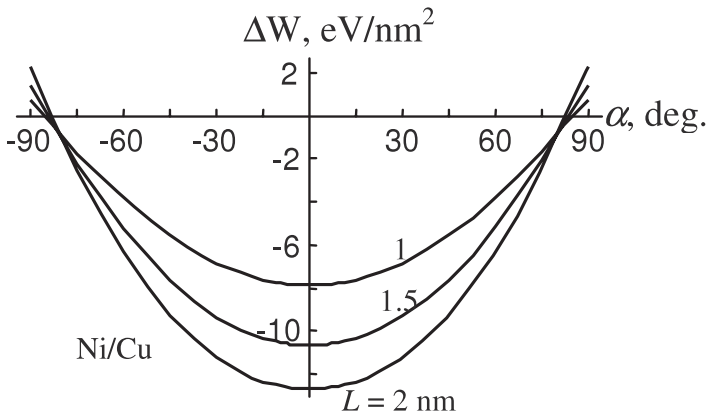

(d)

Figure 5. Dependences of the energy difference $\Delta W$ on angle $\alpha$ (which specifies disclination dipole geometry associated with the direction of grain boundary sliding), for various values of disclination dipole size $L$, in film/substrate systems (a) AlN/6H-SiC, (b) GaN/6H-SiC, (c) $3 \mathrm{C}-\mathrm{SiC} / \mathrm{Si}$ and $(\mathrm{d}) \mathrm{Ni} / \mathrm{Cu}$.

of the disclination dipole is energetically favorable until the moment at which the bottom disclination reaches the filmsubstrate interface. In addition, in real nanocrystalline films, the disclination dipole size $L$ cannot exceed the grain size. (This limitation is related to the geometry of interfacial sliding (figure 1(b))). Also, if the size $L$ of a disclination dipole is 
large enough, a nanocrack can nucleate at this dipole [27]. The disclination dipole with a nanocrack in its vicinity stops being a source of stresses compensating for, in part, the mismatch stresses.

The character of the dependences $\Delta W(L)$ plotted for comparatively thick films is qualitatively similar to that of the dependences $\Delta W(L)$ (figure 3) characterizing thin films with the thickness $H=30 \mathrm{~nm}$, for all the film/substrate systems under consideration, except for 3C-SiC/Si. For instance, figure 4 presents the dependences $\Delta W(L)$ in the case of films specified by the thickness $H=500 \mathrm{~nm}$. All the other parameters of the films are the same as for the thin films (having $H=30 \mathrm{~nm}$ ) described in the two previous paragraphs. The dependences $\Delta W(L)$ shown in figure 4 have minimums, for all the considered film/substrate systems, including 3C-SiC/Si. Thus, with the increase in film thickness in the $3 \mathrm{C}-\mathrm{SiC} / \mathrm{Si}$ system, unlimited expansion of misfit disclination dipoles is replaced by the formation of dipoles with equilibrium sizes.

Figure 5 shows the dependences of the energy difference $\Delta W$ on the angle $\alpha$, for AlN/6H-SiC (figure $5(\mathrm{a})$ ), GaN/6H$\mathrm{SiC}$ (figure 5(b)), 3C-SiC/Si (figure $5(\mathrm{c})$ ) and $\mathrm{Ni} / \mathrm{Cu}$ (figure 5(d)), at the following values of the dipole size: $L=1$, 1.5 and $2 \mathrm{~nm}$. In doing so, in our calculations, we have used the following values of other parameters of the defect structure (figure 2(a)): $p=30 \mathrm{~nm}, H=30 \mathrm{~nm}, h=H / 2 ; \omega=10^{\circ}$, for $\mathrm{AlN} / 6 \mathrm{H}-\mathrm{SiC}$; $-20^{\circ}$, for $\mathrm{GaN} / 6 \mathrm{H}-\mathrm{SiC}$ and $3 \mathrm{C}-\mathrm{SiC} / \mathrm{Si}$; and $20^{\circ}$, for $\mathrm{Ni} / \mathrm{Cu}$. As it follows from figure 5, the generation of disclination dipoles due to interfacial sliding is most favorable at the grain boundaries perpendicular to the film-substrate interface $\left(\alpha=0^{\circ}\right)$.

Figure 6 presents the dependences of the equilibrium value $L_{\mathrm{e}}^{\mathrm{ar}}$ of the disclination dipole size on the angle $\alpha$, in the cases of AlN/6H-SiC (figure 6(a)), GaN/6H-SiC (figure 6(b)) and $\mathrm{Cu} / \mathrm{Ni}$ (figure 6(c)), for $p=30 \mathrm{~nm}, H=30 \mathrm{~nm}, h=H / 2$ and various values of the disclination strength $\omega$. Since the defect configuration under consideration is symmetric relative to the plane $\alpha=0^{\circ}$, only the angle interval $0^{\circ} \leqslant \alpha \leqslant 90^{\circ}$ is shown in figure 6. The dependences $L_{\mathrm{e}}^{\mathrm{ar}}(\alpha)$ (figure 6) indicate that the equilibrium size $L_{\mathrm{e}}^{\mathrm{ar}}$ increases with decreasing disclination strength $\omega$. Also, at certain values of $\alpha$ the dependences $L_{\mathrm{e}}^{\mathrm{ar}}(\alpha)$ have maximums (figure 6). In the case of $3 \mathrm{C}-\mathrm{SiC} / \mathrm{Si}$ film/substrate system with $H=30 \mathrm{~nm}$, the function $L_{\mathrm{e}}^{\mathrm{ar}}(\alpha)$ is not definite. The absence of the equilibrium finite value of disclination dipole size corresponds to the model situations where the unlimited expansion (increase in $L$ ) of the disclination dipole is energetically favorable. This behavior is caused by the very large misfit $(|f|=20 \%)$ of the system 3C$\mathrm{SiC} / \mathrm{Si}$, greatly exceeding in magnitude the misfit parameters $(|f|=0.9-2.6 \%)$ that characterize the systems AlN/6H-SiC, $\mathrm{GaN} / 6 \mathrm{H}-\mathrm{SiC}$ and $\mathrm{Ni} / \mathrm{Cu}$.

Finally, note that, for the systems under consideration and the values of $p$ and $h$ specified in our analysis $(p=30 \mathrm{~nm}$, $h=15 \mathrm{~nm}$ ), the basic contributions to the total energy $\Delta W$ are provided by both the energy $E_{\text {int }}^{\Delta-\Delta}$ of the interaction between the disclination dipoles and the energy $E_{\mathrm{int}}^{\Delta-f}$ that characterizes the interaction of the disclination dipoles with the misfit stress. The contribution of the proper energy $E_{\text {self }}^{\Delta}$ of the disclination dipoles to the total energy $\Delta W$ is comparatively small. (a)

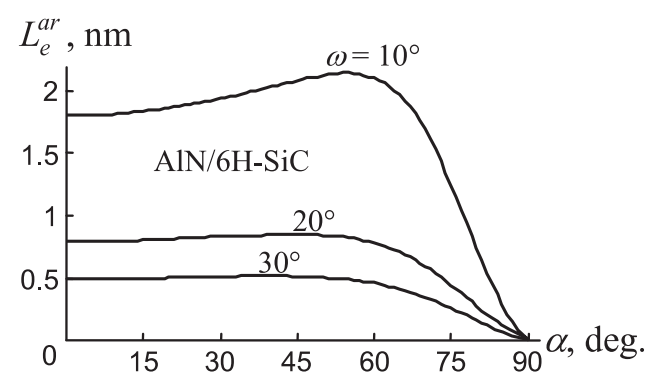

(b)

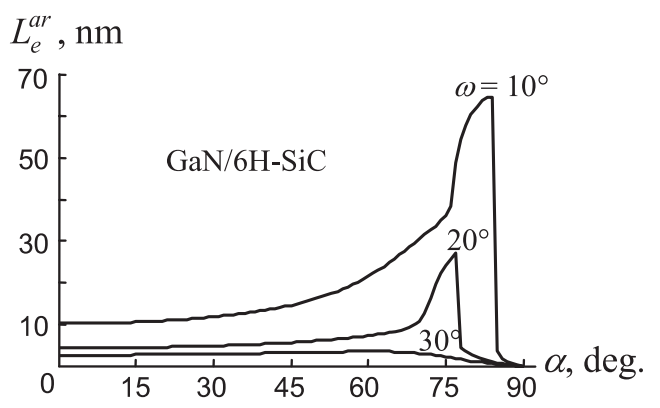

(c)

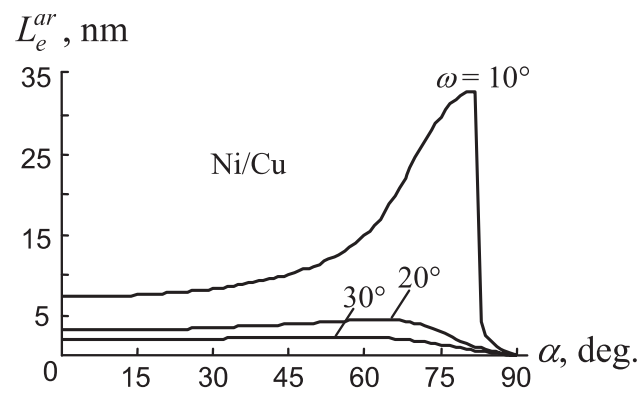

Figure 6. Dependences of equilibrium value $L_{\mathrm{e}}^{\mathrm{ar}}$ of disclination dipole size on angle $\alpha$, for various values of disclination strength $\omega$, in film/substrate systems (a) AlN/6H-SiC, (b) GaN/6H-SiC and (c) $\mathrm{Ni} / \mathrm{Cu}$.

\section{Concluding remarks}

To summarize, the results of our model calculations in the example cases of the nanocrystalline film/substrate systems GaN/6H-SiC, AlN/6H-SiC, 3C-SiC/Si and Ni/Cu show that interfacial sliding accompanied by the formation of wedge disclination dipoles is energetically favorable in these systems in wide ranges of their parameters. This allows us to conclude that the interfacial sliding can serve as a special relaxation mechanism effectively contributing to release of mismatch stresses in nanocrystalline films. At the same time, other stress relaxation mechanisms can effectively operate and contribute to release of mismatch stresses in nanocrystalline films.

Note that the action of the interfacial sliding as a stress relaxation mechanism is inherent to nanocrystalline films, but does not contribute significantly to stress relaxation in conventional coarse-grained and single crystalline films. This is because the formation of disclination dipoles due to the interfacial sliding occurs at triple junctions of grain boundaries, 
whose number is very large in the nanocrystalline matter, in contrast to conventional coarse-grained polycrystals and single crystals. Thus, the stress relaxation due to interfacial sliding in nanocrystalline films represents a specific manifestation of the nanoscale and interface effects in these films.

\section{Acknowledgments}

The work was supported, in part, by the Russian Federal Agency of Science and Innovations (Contract 02.513.11.3190 of the Program 'Industry of Nanosystems and Materials' and grants MK-5122.2007.1, MK-1702.2008.1 and NSh2405.2008.1), the Russian Foundation of Basic Research (grant 08-01-00225-a), the National Science Foundation (grant CMMI \#0700272), the Committee on Science and Higher Schools of the St Petersburg Government and the Russian Academy of Sciences Program 'Structural Mechanics of Materials and Construction Elements'.

\section{References}

[1] Babkevich A Yu, Cowley R A, Mason N J, Weller S and Stunault A 2002 J. Phys.: Condens. Matter 14 13505-28

[2] Lavagne S, Levade C and Vanderschaeve G 2002 J. Phys.: Condens. Matter 14 13291-8

[3] Ovid'ko I A 2002 Phys. Rev. Lett. 88046103

[4] Ovid'ko I A, Sheinerman A G and Skiba N V 2003 J. Phys.: Condens. Matter 15 1173-81

[5] Yastrubchak O, Wosinski T, Domagala J Z, Lusakowska E, Figielski T, Pecz B and Toth A L 2004 J. Phys.: Condens. Matter 16 S1-8

[6] Hakkarainen T, Toivonen J, Koskenvaara H, Sopanen M and Lipsanen H 2004 J. Phys.: Condens. Matter 16 S3009-26

[7] Ovid'ko I A and Sheinerman A G 2006 Adv. Phys. 55 627-89

[8] Siwach P K, Singh H K and Srivastava O N 2006 J. Phys.: Condens. Matter 18 9783-94

[9] Ovid'ko I A and Sheinerman A G 2007 J. Phys.: Condens. Matter 19056008

[10] Muduli P K, Bose S K and Budhani R C 2007 J. Phys.: Condens. Matter 19226204

[11] Tjong S C and Chen H 2004 Mater. Sci. Eng. R 45 1-88

[12] Cavaleiro A and De Hosson J Th M (ed) 2006 Nanostructured Coatings (New York: Springer Science + Business Media LLC)

[13] Tang C J, Neves A J, Fernandes A J S, Gracio J and Carmo M C 2007 J. Phys.: Condens. Matter 19386236

[14] Cheng Q J, Long J D, Chen Z and Xu S 2007 J. Phys. D: Appl. Phys. 40 2304-7

[15] Kumar K U M, Brahma R, Krishna M G, Bhatnagar A K and Dalba G 2007 J. Phys.: Condens. Matter 19496208

[16] Sadananda K and Holz R L 2000 Nanostructured Films and Coatings ed G-M Chow, I A Ovid'ko and T Tsakalakos (Dordrecht: Kluwer) pp 283-95

[17] Kabacoff L T 2000 Nanostructured Films and Coatings ed G-M Chow, I A Ovid'ko and T Tsakalakos (Dordrecht: Kluwer) pp 373-7

[18] Akbari A, Riviere J P, Templier C, Le Bourhis E and Abadias G 2007 Rev. Adv. Mater. Sci. 15 111-7
[19] Meyers M A, Mishra A and Benson D J 2006 Prog. Mater. Sci. $51427-556$

[20] Dao M, Lu L, Asaro R J, De Hosson J T M and Ma E 2007 Acta Mater. 55 4041-65

[21] Koch C C, Ovid'ko I A, Seal S and Veprek S 2007 Structural Nanocrystalline Materials: Fundamentals and Applications (Cambridge: Cambridge University Press)

[22] Mukherjee A K 2002 Mater. Sci. Eng. A 322 1-22

[23] Van Swygenhoven H and Derlet P M 2001 Phys. Rev. B 64224105

[24] Padmanabhan K A and Gleiter H 2004 Mater. Sci. Eng. A $38128-38$

[25] Gutkin M Yu, Ovid'ko I A and Skiba N V 2004 Acta Mater. 52 1711-20

[26] Xu X, Nishimura T, Hirosaki N, Xie R J, Yamamoto Y and Tanaka H 2006 Acta Mater. 54 255-62

[27] Ovid'ko I A and Sheinerman A G 2007 Appl. Phys. Lett. 90171927

[28] Ovid'ko I A and Sheinerman A G 2008 Phys. Rev. B 77054109

[29] Romanov A E and Vladimorov V I 1992 Dislocation in Solids vol 9, ed F R N Nabarro (Amsterdam: North-Holland) pp 191-302

[30] Kleman M and Friedel J 2008 Rev. Mod. Phys. 80 61-115

[31] Murayama M, Howe J M, Hidaka H and Takaki S 2002 Science 295 2433-5

[32] Klimanek P, Klemm V, Romanov A E and Seefeldt M 2001 Adv. Eng. Mater. 3 877-84

[33] Klemm V, Klimanek P and Motylenko M 2002 Solid State Phenom. 87 57-72

[34] Sutton A P and Balluffi R W 1995 Interfaces in Crystalline Materials (Oxford: Clarendon)

[35] Valiev R Z 2004 Nat. Mater. 3 511-6

[36] Langdon T G 2006 J. Mater. Sci. 46 597-609

[37] Aita C R and Tait W S 1992 Nanostruct. Mater. 1 269-82

[38] Bondar V 1999 Phys. Status Solidi a 179 635-8

[39] Szmidt J, Sokołowska A, Olszyna A, Werbowy A and Pawłowski P 1999 Diamond Relat. Mater. 8 391-7

[40] Drüsedau T P and Bläsing J 2000 Thin Solid Films 377/378 27-31

[41] Cibert C, Tétard F, Djemia P, Champeaux C, Catherinot A and Tétard D 2004 Superlatt. Microstruct. 36 4-6

[42] Kulikovsky V, Vorlíček V, Boháč P, Stranyánek M, Čtvrtlík R, Kurdyumov A and Jastrabik L 2008 Surf. Coat. Technol. 202 1738-45

[43] Shen Y F, Xue W Y, Wang Y D, Liu Z Y and Zuo L 2008 Surf. Coat. Technol. 202 5140-5

[44] Wang L, Zhang J, Gao Y, Xue Q, Hu L and Xu T 2006 Scr. Mater. 55 657-60

[45] Wright A F 1997 J. Appl. Phys. 82 2833-9

[46] Kim M, Ohta J, Kobayashi A, Fujioka H and Oshima M 2008 Phys. Status Solidi (RRL) 2 13-5

[47] Rawn C J and Chaudhuri J 2000 Advances in X-ray Analysis vol 43 (Denver: JCPDS-International Central for Diffraction Data) pp 338-43

[48] Ding Z, Zhou S and Zhao Y 2007 Phys. Rev. B 70184117

[49] Shoji A, Nakamura M, Mitikami K, Isshiki T, Ohshima S and Nishino S 2005 Mater. Sci. Forum 483-485 221-4

[50] Hirth J P and Lothe J 1982 Theory of Dislocation (New York: Wiley)

[51] Asaro R J and Suresh S 2005 Acta Mater. 53 3369-82 